Frederike Schmidt, Miryam Müller, Johannes Prox, Philipp Arnold, Caroline Schönherr, Claudia Tredup, Petra Minder, Henriette Ebsen, Ottmar Janssen, Wim Annaert, Claus Pietrzik, Dirk Schmidt-Arras, Erwin E. Sterchi and Christoph Becker-Pauly*

\title{
Tetraspanin 8 is an interactor of the metalloprotease meprin $\beta$ within tetraspanin-enriched microdomains
}

DOI 10.1515/hsz-2016-0126

Received January 29, 2016; accepted May 4, 2016; previously published online May 13, 2016

\begin{abstract}
Meprin $\beta$ is a dimeric type I transmembrane protein and acts as an ectodomain sheddase at the cell surface. It has been shown that meprin $\beta$ cleaves the amyloid precursor protein (APP), thereby releasing neurotoxic amyloid $\beta$ peptides and implicating a role of meprin $\beta$ in Alzheimer's disease. In order to identify non-proteolytic regulators of meprin $\beta$, we performed a split ubiquitin yeast two-hybrid screen using a small intestinal cDNA library. In this screen we identified tetraspanin 8 (TSPAN8) as interaction partner for meprin $\beta$. As several members of the tetraspanin family were described to interact with metalloproteases thereby affecting their localization and/or activity, we hypothesized similar functions of TSPAN8 in the regulation of meprin $\beta$. We employed cell biological methods to confirm direct binding of TSPAN8 to meprin $\beta$. Surprisingly, we did not observe an effect of
\end{abstract}

\footnotetext{
*Corresponding author: Christoph Becker-Pauly, Institute of Biochemistry, University of Kiel, Otto-Hahn-Platz 9, D-24118 Kiel, Germany, e-mail: cbeckerpauly@biochem.uni-kiel.de Frederike Schmidt, Miryam Müller, Johannes Prox and Dirk Schmidt-Arras: Institute of Biochemistry, University of Kiel, Otto-Hahn-Platz 9, D-24118 Kiel, Germany

Philipp Arnold: Anatomical Institute, University of Kiel, D-24118 Kiel, Germany

Caroline Schönherr and Claus Pietrzik: Institute of Pathobiochemistry, University Medical Centre of the Johannes Gutenberg University of Mainz, D-55128 Mainz, Germany Claudia Tredup: Pharmazentrum Frankfurt, Institut für Allgemeine Pharmakologie und Toxikologie, Klinikum der Goethe-Universität Frankfurt am Main, D-60590 Frankfurt am Main, Germany Petra Minder: Department of Molecular and Experimental Medicine, The Scripps Research Institute, La Jolla, CA 92037, USA Henriette Ebsen and Ottmar Janssen: Institute of Immunology, University of Kiel, University Hospital Schleswig-Holstein Campus Kiel, D-24105 Kiel, Germany

Wim Annaert: Center for Human Genetics, KU Leuven and VIB Center for the Biology of Disease, B-3000 Leuven, Belgium

Erwin E. Sterchi: Institute of Biochemistry and Molecular Medicine, University of Bern, CH-3012 Bern, Switzerland
}

TSPAN8 on the catalytic activity of meprin $\beta$ nor on the specific cleavage of its substrate APP. However, both proteins were identified as present in tetraspanin-enriched microdomains. Therefore we hypothesize that TSPAN8 might be important for the orchestration of meprin $\beta$ at the cell surface with impact on certain proteolytic processes that have to be further identified.

Keywords: APP; plasma membrane; protein-protein interaction; proteolysis; TEMs.

\section{Introduction}

Meprin $\beta$ belongs to the astacin family of metalloproteases and is synthesized as a dimeric type I transmembrane protein (Sterchi et al., 2008). Upon removal of its propeptide by serine proteases, such as kallikrein-related peptidases, meprin $\beta$ gains full catalytic activity (Ohler et al., 2010). Recently, we identified matriptase-2 as a specific activator of membrane-bound meprin $\beta$ (Jäckle et al., 2015). At the cell surface meprin $\beta$ acts as an ectodomain sheddase (Arolas et al., 2012) and processes the amyloid precursor protein (APP) at the $\beta$-secretase site, reminiscent to the $\beta$-site amyloid precursor protein cleaving enzyme 1 (BACE1) (Bien et al., 2012). Upon additional cleavage of the $\gamma$-secretase, short amyloid $\beta(A \beta)$ peptides are released prone to form aggregates, which are assumed to cause neuronal death in Alzheimer's patients (Perneczky et al., 2014). However, the contribution of meprin $\beta$ in Alzheimer's disease has to be further elucidated. Meprin $\beta$ can also be shed from the cell surface by a disintegrin and metalloproteinases (ADAMs) (Hahn et al., 2003; Jefferson et al., 2013) and is able to cleave soluble extracellular substrates such as mucin 2 (MUC2). MUC2 is important for normal barrier function in the small intestine and has to be released into the lumen via proteolysis (Schütte et al., 2014). Meprin $\beta$ has further been characterized as procollagen processing enzyme leading to fibril formation and collagen deposition (Kronenberg et al., 2010; Broder et al., 2013). In Fra-2 transgenic mice, which develop cardiac hypertrophy and lung fibrosis, meprin $\beta$ was shown to be the most up-regulated gene in fibrotic 
lung tissue (Biasin et al., 2014). As up-regulation of meprin $\beta$ may play an important role in the onset of certain pathologies, the regulation of its activity and its shedding has to be tightly controlled.

In recent years several members of the tetraspanin family were identified as regulatory proteins for different proteases (Yanez-Mo et al., 2011). Tetraspanins possess four membrane helices: the short $\mathrm{N}$ - and C-terminal tails are cytoplasmic whereas a small and a large loop are formed extracellularly. The latter includes highly conserved cysteines, forming two to four disulfide bonds, thereby stabilizing the three-dimensional structure of this region, which is thought to be involved in tetraspanin homodimerization and client protein interaction (Charrin et al., 2014). Tetraspanins form higher-order lipid-protein membrane complexes termed tetraspanin-enriched microdomains (TEMs) that differ from lipid rafts. To build up these clusters tetraspanins interact not only with other tetraspanins, but also with further transmembrane and cytosolic proteins (Charrin et al., 2009; Yanez-Mo et al., 2009). TEMs represent specialized structures providing a signaling platform by which tetraspanins, also called 'molecular facilitators', are involved in a variety of different biological processes (Hemler, 2003, 2005). It has been shown that tetraspanins modulate the cellular localization and activation of metalloproteases upon binding (Gutierrez-Lopez et al., 2011; Haining et al., 2012; Prox et al., 2012).

To better understand the mechanisms that control shedding and proteolytic activity of meprin $\beta$, we performed a yeast two-hybrid screen to identify potential interacting proteins that might represent regulators of meprin $\beta$. We identified the tetraspanin family member Tetraspanin 8 (TSPAN8) and investigated its impact on meprin $\beta$ localization and activity.

\section{Results}

\section{TSPAN8 specifically interacts with meprin $\beta$ at the cell surface}

To identify regulatory non-proteolytic interacting proteins for human meprin $\beta$ a split-ubiqutin yeast two-hybrid screen was performed. As meprin $\beta$ is strongly expressed in the intestinal tract in humans, a small intestine cDNA library was chosen as prey. In this screen TSPAN8 was identified as putative interaction partner for meprin $\beta$ (Figure 1A; complete list of interaction partners is given in Supplementary Table 1). We further analyzed the interaction between TSPAN8 and meprin $\beta$, because several tetraspanins were previously shown to interact with metalloproteases, thereby influencing their activity or localization (Gutierrez-Lopez et al., 2011; Haining et al., 2012; Prox et al., 2012).

To confirm the protein-protein interaction between meprin $\beta$ and TSPAN8 in mammalian cells we performed a luciferase-based protein complementation assay (Cassonnet et al., 2011). Meprin $\beta$ and TSPAN8 were each C-terminally fused to one inactive half of the Gaussia princeps luciferase (Figure 1B). HEK293T cells were transiently transfected with these constructs and the corresponding cell lysates were used for determination of proximitymediated luciferase activity reconstitution through measurement of coelenterazine-mediated light emission. The normalized luciferase ratio (NLR) indicates the extent of protein-protein interaction and an NLR greater than 3.5 implies specific interaction of two proteins (Cassonnet et al., 2011). The NLR of meprin $\beta$ and TSPAN8 was between 50 and 60, thus confirming the interaction observed in the yeast two-hybrid screen. As meprin $\beta$ as well as tetraspanins are known to form homodimers (Kovalenko et al., 2004; Arolas et al., 2012), we included this in the complementation assay as positive controls. Homodimers of meprin $\beta$ or TSPAN8 showed an NLR of 120-140 (Figure 1B).

To further assess in which cellular compartment the interaction between meprin $\beta$ and TSPAN8 takes place, a red fluorescent protein (RFP) dimerization assay was performed (Figure 1C). This protein complementation technique is based on the ability of specific RFP monomers to show a 10 -fold increase in fluorescence when dimerization occurs (Alford et al., 2012). HEK293T cells were transiently transfected with meprin $\beta$ and TSPAN8, both C-terminally fused to one RFP monomer each. The RFP dimerization was evaluated by fluorescence microscopy $48 \mathrm{~h}$ post transfection. Red fluorescence was detectable at the plasma membrane of HEK293T cells expressing both RFP-fused proteins, corroborating specific interaction of TSPAN8 and meprin $\beta$. Controls that were transfected with one fusion construct and a RFP monomer alone showed no red fluorescence. Taken together, direct interaction of TSPAN8 and meprin $\beta$ was detected in the yeast-two hybrid screen as well as in mammalian cells by a luciferase-based complementation assay and the RFP dimerization assay.

\section{TSPAN8 has no direct influence on the proteolytic activity of meprin $\beta$}

To further investigate a potential effect of TSPAN8 on meprin $\beta$ activity, we used HEK293T cells expressing 
A

\begin{tabular}{llllll} 
Prey-ID & Frame & Uniprot ID & Protein name (description) & Expectation \\
\hline PPHb-mat_prey_003 & 1 & P19075 & Tetraspanin-8 OS=Homo sapiens GN=TSPAN8 PE=1 SV=1 & $3 E-61$ \\
PPHb-mat_prey_040 & 1 & P19075 & Tetraspanin-8 OS=Homo sapiens GN=TSPAN8 PE=1 SV=1 & $2 E-72$ \\
PPHb-mat_prey_127 & 1 & P19075 & Tetraspanin-8 OS=Homo sapiens GN=TSPAN8 PE=1 SV=1 & $8 E-88$
\end{tabular}

B
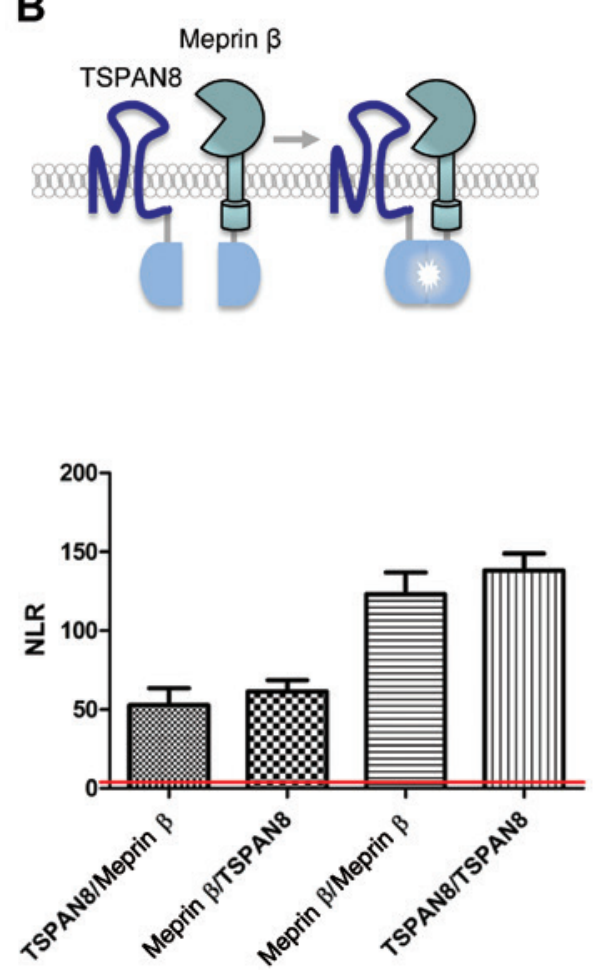

C
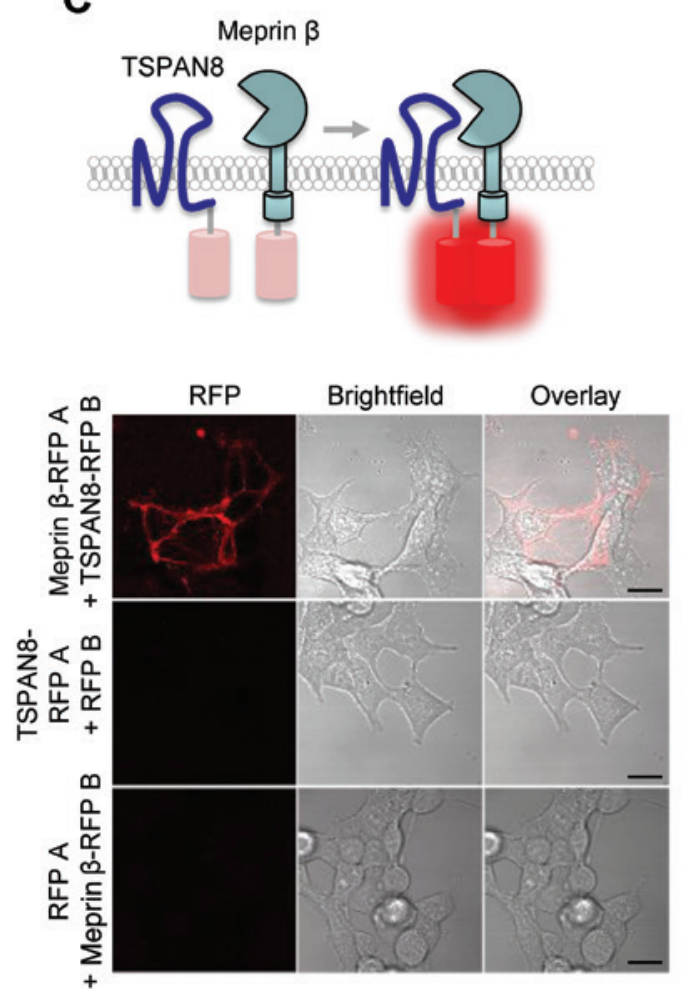

Figure 1: TSPAN8 is a novel interaction partner of the metalloprotease meprin $\beta$.

(A) A split-ubiquitin yeast two-hybrid screen was performed using human meprin $\beta$ (PPhb-mat) as bait and a human small intestinal cDNA library as prey. Several proteins were identified as putative interaction partners for meprin $\beta$, among them being tetraspanin 8 (TSPAN8) which was positively sequenced in frame. (B) Gaussia princeps luciferase-based protein complementation assay confirmed interaction between TSPAN8 and meprin $\beta$. TSPAN8 and meprin $\beta$ were each fused to one half of inactive $G$. princeps luciferase (light blue). Interaction of both proteins led to reconstitution of the luciferase and its activity was analyzed via the conversion of coelenterazine. The extent of protein interaction is indicated by the normalized luciferase ration (NLR). Specific interaction exists when NLR is $>3.5$ (red line). $n=3$. (C) Red fluorescent protein (RFP) dimerization assay showed interaction of TSPAN8 and meprin $\beta$ at the cell surface of HEK293T cells. Both proteins were fused to an RFP monomer (RFP A and RFP B) and upon interaction of meprin $\beta$ and TSPAN8 RFP dimerized leading to a 10-fold increase in fluorescence (upper panel). Controls where only one protein of interest was fused to RFP showed no red fluorescence (middle and lower panel). Scale bar $=20 \mu \mathrm{m}$.

TSPAN8 in a doxycycline-inducible manner. For further analysis of the potential interaction site we focused on the large extracellular loop (LEL) of TSPAN8 since this region is thought to mediate interaction with client proteins. Interestingly, in the yeast two-hybrid screen TSPAN8 was only identified as an interaction partner for active meprin $\beta$ and not for its proform. We therefore induced two point mutations (E133A and E135A) in a specific region of the LEL of TSPAN8 that shows sequence similarities to the propeptide of meprin $\beta$ (Supplementary Figure 1A, B and C).

To analyze whether TSPAN8 has a negative impact on the proteolytic activity of meprin $\beta$, a specific meprin $\beta$ activity assay was performed (Figure $2 \mathrm{~A})$. We used the quenched fluorogenic peptide (mca)-EDEDED-(K- $\varepsilon$-dnp) (Broder et al., 2013; Jäckle et al., 2015) to evaluate the 
A

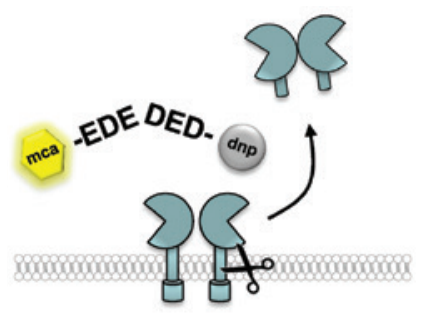

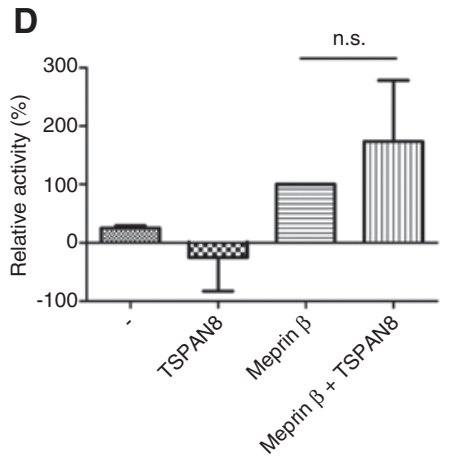

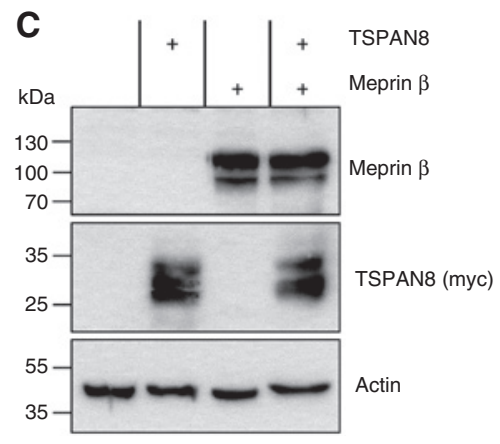

B

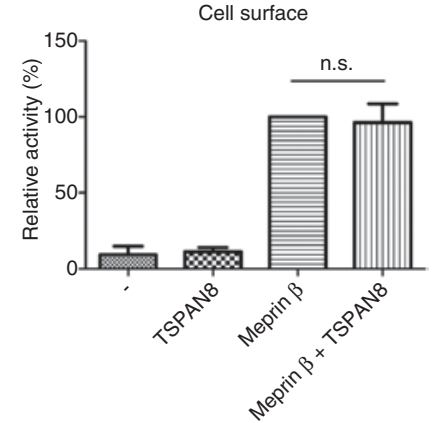

E

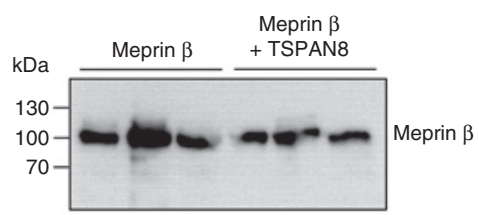

Figure 2: Influence of TSPAN8 on meprin $\beta$ activity.

(A) Proteolytic activity of membrane bound or shed soluble meprin $\beta$ was analyzed using the specific quenched fluorogenic peptide (mca)EDEDED-(K- $\varepsilon$-dnp). Intensity of fluorescence was measured at $405 \mathrm{~nm}$ with an excitation at $320 \mathrm{~nm}$ every $30 \mathrm{~s}$ for $2 \mathrm{~h}$ at $37^{\circ} \mathrm{C}$. dnp: $2,4-\mathrm{dini}-$ trophenol; mca: (7-methyloxycoumarin-4-yl) acetyl. (B) TSPAN8 does not influence the proteolytic activity of meprin $\beta$ at the plasma membrane. HEK293T cells were transfected with meprin $\beta$ and TSPAN8 expression was induced. Relative activity of meprin $\beta$ at the cell surface is shown. $n=7$. (C) Representative Western blot analysis of meprin $\beta$ and TSPAN8 expression in cells that were used for the activity assay. Actin served as loading control. (D) TSPAN8 has no effect on the shedding of meprin $\beta$ from the cell surface. HEK293T cells were transfected with meprin $\beta$ and TSPAN8 expression was induced. Relative activity of shed meprin $\beta$ within the cell culture medium is shown. $n=3$. (E) Western blot analysis of shed meprin $\beta$ in the supernatant of transfected HEK293T cells from three independent experiments.

activity of meprin $\beta$ at the surface of HEK293T cells in presence or absence of TSPAN8. Untransfected cells or cells expressing only TSPAN8 upon doxycycline induction showed almost no meprin $\beta$ activity, whereas a strong increase in fluorescence was measured in meprin $\beta$ transfected cells. Interestingly, the relative activity of meprin $\beta$ was not altered when coexpressing TSPAN8, indicating that TSPAN8 has no direct effect on the proteolytic activity of meprin $\beta$ (Figure $2 \mathrm{~B}, \mathrm{C}$ ).

We then cotransfected the TSPAN8_E133A_E135A mutant and meprin $\beta$ into HEK293T cells and again measured the activity of meprin $\beta$ at the cell surface. We did not observe any difference in meprin $\beta$ activity in the presence of TSPAN8 or its mutant (Supplementary Figure 1D). This led us to the conclusion that the two glutamate residues in the LEL of TSPAN8 are not directly binding the active site of meprin $\beta$ and do not influence meprin $\beta$ activity.

Additionally, we analyzed the culture medium of HEK293T cells with regard to meprin $\beta$ activity to investigate possible alterations in the ectodomain shedding of meprin $\beta$ from the cell surface. However, we did not observe a significant difference in meprin $\beta$ activity in presence or absence of TSPAN8 (Figure 2D, E).

\section{Processing of APP by meprin $\beta$ in presence of TSPAN8}

As TSPAN8 has no impact on general meprin $\beta$ activity, we wanted to analyze whether TSPAN8 has an impact on the accessibility of proteinogenic substrates for meprin $\beta$. For this, we chose APP as a well described substrate readout for meprin $\beta$. Previously, meprin $\beta$ was shown to cleave APP at the $\beta$-secretase site, thereby releasing short A $\beta$ peptides (Bien et al., 2012) (Figure 3A). Here, we used secreted $A \beta$ peptides as readout for meprin $\beta$ activity. As expected, when coexpressing meprin $\beta$ and APP high levels of $\mathrm{A} \beta$ peptides were generated (Figure $3 \mathrm{~B}$ ). 
A

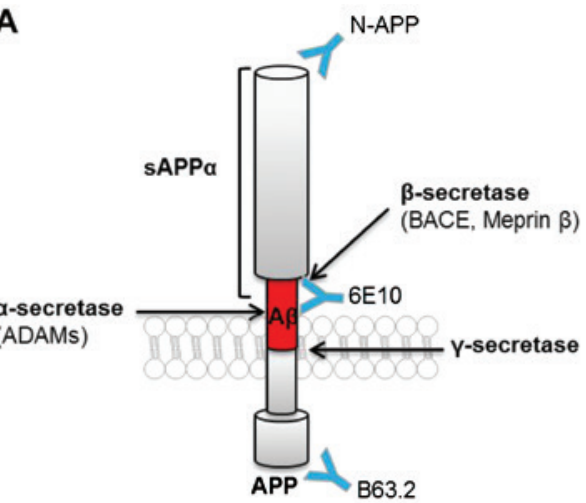

B

$$
\text { C }
$$
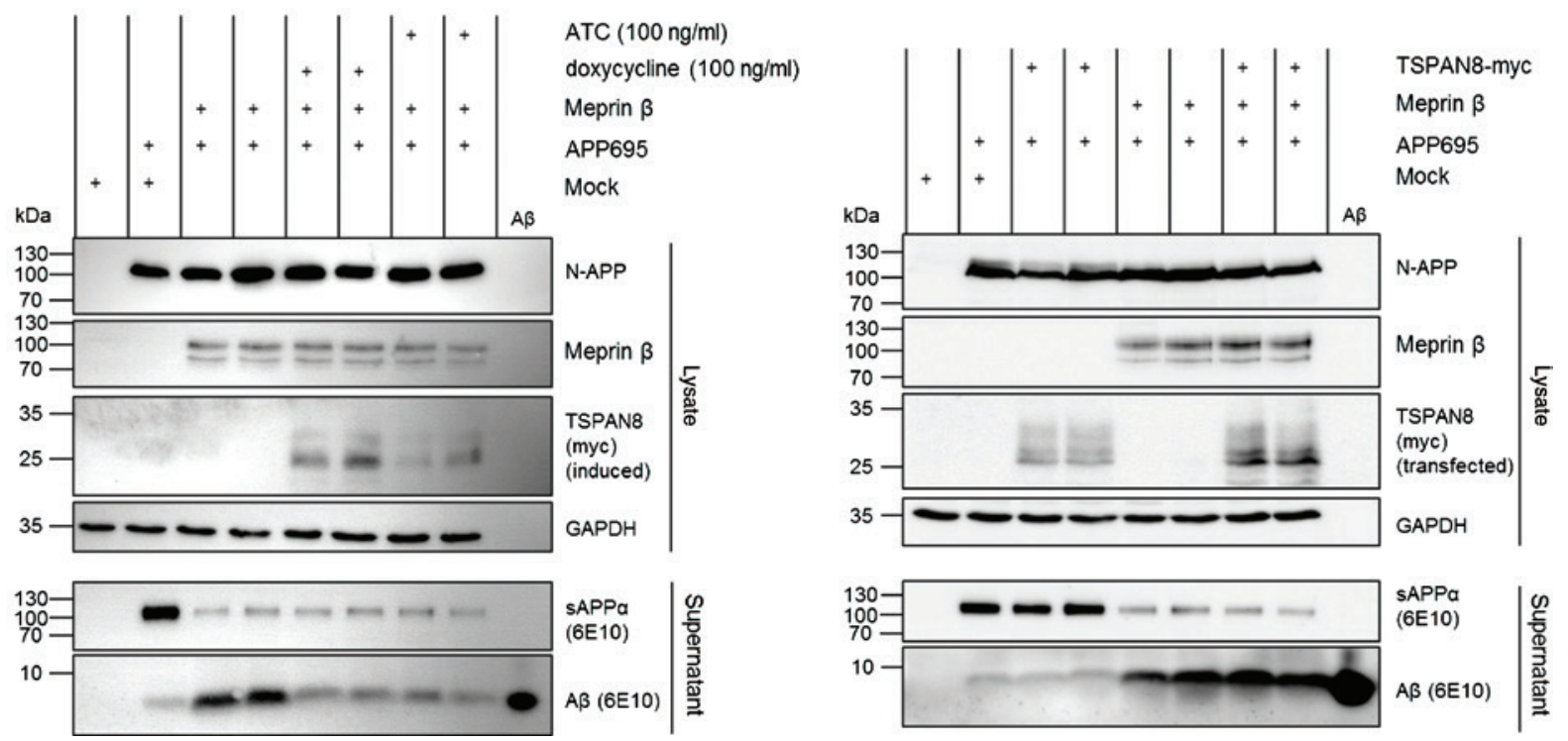

Figure 3: Processing of APP in the presence of TSPAN8.

(A) The amyloid precursor protein (APP) is a type I transmembrane protein. APP is processed by a $\beta$ - and subsequently by $\gamma$-secretase thereby releasing amyloid $\beta(A \beta)$ peptides (red). Alternatively, $\alpha$-secretase cleavage prevents A $\beta$ production via generation of soluble APP (sAPP $\alpha$ ). Three different antibodies directed against APP were used in this study (blue Y). (B) Analysis of APP cleavage by meprin $\beta$ upon induction of TSPAN8 expression. HEK293T cells were transfected with meprin $\beta$ and APP, TSPAN8 expression was induced with either anhydrotetracycline (ATC) or doxycycline. Meprin $\beta$ expression yielded in high amounts of A $\beta$ peptides and subsequent decrease of sAPP $\alpha$ levels. In the presence of TSPAN8 A $\beta$ generation was diminished but no increase in SAPP $\alpha$ was detectable. (C) Analysis of APP cleavage by meprin $\beta$ upon transient transfection of TSPAN8. HEK293T cells were transfected with meprin $\beta$, APP and TSPAN8. Again, processing of APP by meprin $\beta$ was accompanied by high $A \beta$ levels and reduction in the amount of SAPP $\alpha$. Under these conditions the presence of TSPAN8 did not alter the generation of $A \beta$ peptides.

Correspondingly, only little soluble APP $\alpha$ (sAPP $\alpha$ ) was present in the supernatant of HEK293T cells, since overexpressed meprin $\beta$ dominates $\beta$-secretase cleavage which competes with or occurs prior to shedding of APP by ADAMs at the $\alpha$-secretase site. Interestingly, induction of TSPAN8 expression by doxycycline or anhydrotetracycline (ATC) treatment resulted in reduced amounts of $\mathrm{A} \beta$. However, the corresponding level of $\mathrm{sAPP} \alpha$ did not increase. In order to analyze whether the reduction in $A \beta$ might be an effect of doxycycline or ATC, we performed a similar experiment but this time transfecting TSPAN8 instead of inducing its expression (Figure 3C). Again, strong $A \beta$ production was present when coexpressing APP and meprin $\beta$. Of note, the presence of TSPAN8 did not result in any changes in $A \beta$ generation concluding that the previous effect was caused by the treatment with doxycycline or ATC. This experiment shows that the presence of TSPAN8 does not change the accessibility of the substrate APP for meprin $\beta$, thus not altering the generation of $A \beta$ peptides. 
A

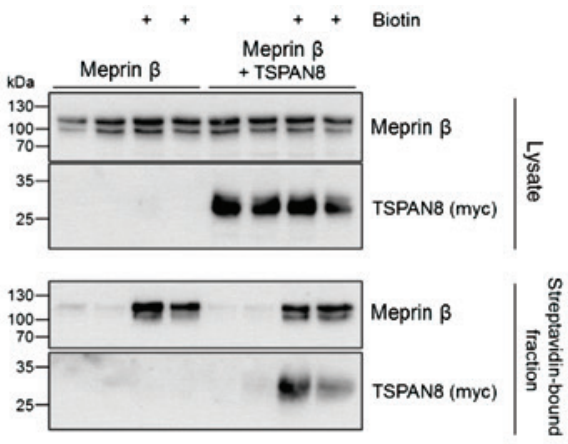

B

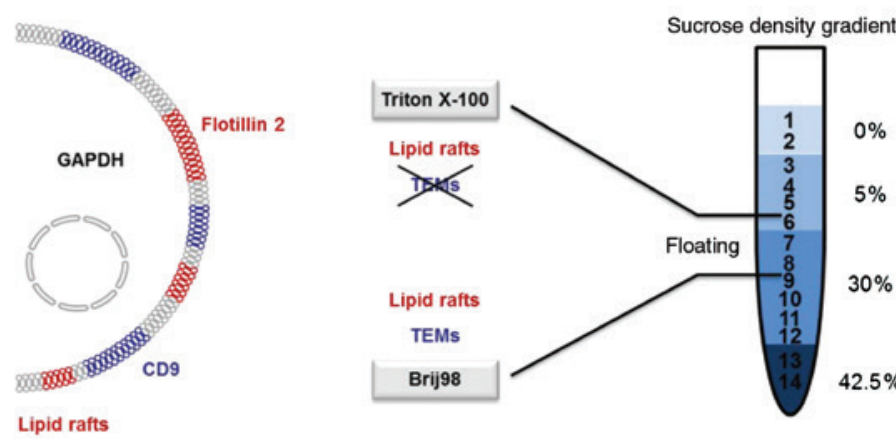

TEMs

C

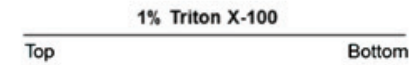

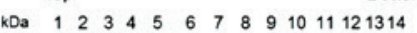
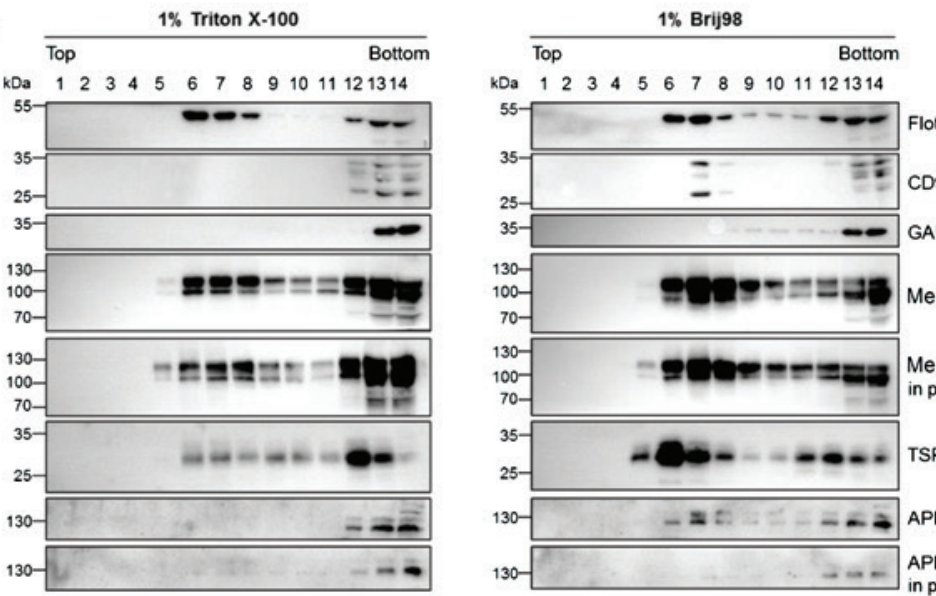

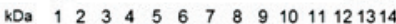
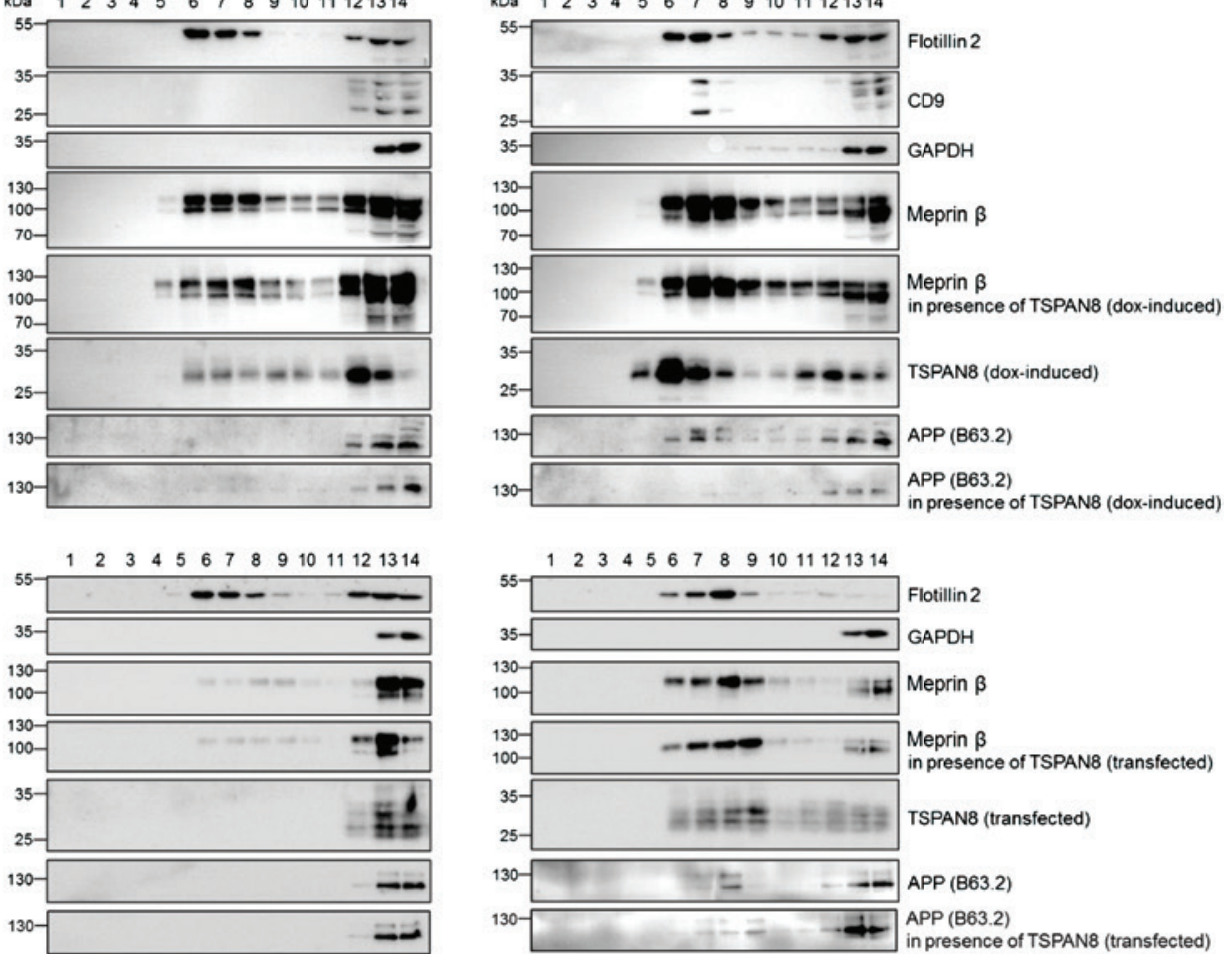

\section{TSPAN8 and meprin $\beta$ colocalize within tetraspanin-enriched microdomains}

As we could exclude a direct effect of TSPAN8 on the catalytic site of meprin $\beta$ or its shedding from the cell surface, we decided to further analyze the cell surface localization of meprin $\beta$ and TSPAN8. We first analyzed the cell surface levels of both meprin $\beta$ and TSPAN8 in the inducible HEK293T cells by primary amine biotinylation of proteins (Figure 4A). Indeed, both proteins, meprin $\beta$ and TSPAN8, 
Figure 4: Distribution of meprin $\beta$, TSPAN8, and APP at the cell surface.

(A) HEK293T cells were transiently transfected with meprin $\beta$ and stable expression of TSPAN8 was induced by doxycycline. Cell surface levels of both proteins were analyzed applying primary amine biotinylation. TSPAN8 as well as meprin $\beta$ were found at the cell surface of HEK cells. TSPAN8 had no effect on the trafficking of meprin $\beta$ to the cell surface. Additionally, no change in meprin $\beta$ maturation was visible in presence of TSPAN8 (upper band: promeprin $\beta$, lower band: active meprin $\beta$ ). (B) Isolation of lipid rafts or tetraspanin-enriched microdomains (TEMs) via sucrose density gradient centrifugation. Flotillin 2 served as marker protein for lipid rafts (red) whereas the tetraspanin CD9 is located in TEMs (blue). Two different detergents were used for cell lysis: Triton X-100 preserves lipid rafts but destroys TEMs whereas with the milder detergent Brij98 both membrane regions stay intact. Discontinuous sucrose density gradient ( $0-42.5 \%$ sucrose) was prepared upon loading the cell lysate at the bottom of the centrifuge tube. After centrifugation TEMs and lipid rafts float within fractions 6-8. (C) HEK293T cells were transfected with meprin $\beta$ and TSPAN8 expression was induced by doxycycline (dox) (upper part). Cells lysates, either prepared with Triton X-100 or Brij98, were loaded on sucrose density gradient. Marker proteins (Flotillin 2, CD9 and GAPDH) showed that the sucrose density gradient worked. Meprin $\beta$ is found in lipid rafts but also in TEMs whereas TSPAN8 is predominantly located within TEMs. Endogenous APP was detected to a higher extent in TEMs than lipid rafts. When the expression of TSPAN8 was induced by doxycycline the signal for APP within TEMs was diminished. To verify this we performed the same experiment but this time transfected TSPAN8 instead of inducing its expression via doxycycline (lower part). Again, APP was predominantly found in TEMs but the presence of TSPAN8 did not alter its distribution.

were detectable in the streptavidin bound fractions and were thus localized at the cell surface. This is in accordance with the observation of the RFP dimerization assay where TSPAN8-meprin $\beta$ interaction was mainly detectable at the cell surface (Figure 1C). TSPAN8 expression did not alter the amount of meprin $\beta$ at the cell surface as we observed equal protein levels in the induced and non-induced cells. Moreover the ratio of pro- and mature meprin $\beta$ did not change when coexpressing TSPAN8, suggesting that TSPAN8 does not influence the maturation of meprin $\beta$. These data fit nicely to the results from the peptide cleavage assays (Figure 2) and confirm that the level and therefore the activity of meprin $\beta$ at the cell surface are unchanged and that an altered shedding of meprin $\beta$ in the presence of TSPAN8 can be excluded.

We then analyzed the submembranous localization of TSPAN8 and meprin $\beta$ by a sucrose density gradient centrifugation of cell lysates (Figure 4B). Tetraspanins were already described to form lipid raft-like membrane fractions called tetraspanin-enriched microdomains (TEMs) (Charrin et al., 2009; Yanez-Mo et al., 2009). We used two different detergents for cell lysis to distinguish between lipid rafts and TEMs. Triton X-100 destroys TEMs but preserves lipid rafts which float in the sucrose density gradient whereas the milder detergent Brij98 enables floating of both membrane fractions. The cell lysates were loaded on discontinuous sucrose gradients and following centrifugation 14 fractions were collected and analyzed by Western blotting.

We first checked different marker proteins to ensure that the sucrose density gradient worked properly (Figure 4C). Flotillin 2 is localized within lipid rafts and therefore was found in the floating region (fractions 6-8) when the cells were lysed with Triton X-100. The TEMs, which are characterized by the presence of the tetraspanin CD9, were also found in the same fractions when treating the cells with Brij98. GAPDH served as a control as it is not associated with these membrane regions and therefore was only found in the high density fractions 12-14.

We analyzed the localization of meprin $\beta$ and demonstrate that meprin $\beta$ is located in lipid rafts. However, we did not observe any change in localization within the membrane when inducing the expression of TSPAN8. Usage of the milder detergent Brij98 revealed that meprin $\beta$ is also found in TEMs, as the amount of meprin $\beta$ increases in the low density fractions 6-8 and decreases in the high density fractions 12-14. Under these conditions TSPAN8 was also present in the fractions corresponding to TEMs. As levels of TSPAN8 are relatively lower in lipid rafts, this indicates that the interaction between meprin $\beta$ and TSPAN8 takes place within the TEMs. Furthermore, we investigated the distribution of the substrate APP. We could not detect endogenous APP in lipid rafts in the presence or absence of TSPAN8. However, APP was found in TEMs in the absence of TSPAN8. Interestingly, when TSPAN8 expression was induced the amount of endogenous APP was reduced in these fractions. This is consistent with the reduced $A \beta$ generation by meprin $\beta$ when TSPAN8 expression was induced by doxycycline or ATC (Figure 3B). As we could already show that the effect on $A \beta$ is not visible any more in the absence of doxycycline, we repeated the sucrose density gradient centrifugation using cells that were cotransfected with meprin $\beta$ and TSPAN8 (Figure 4C lower part). We confirmed similar distribution of meprin $\beta$ within the cell membrane as described above. When analyzing endogenous APP we could show again that APP is found only to very little extent in lipid rafts in these HEK cells. APP is rather located within TEMs, as there is 
a strong signal for APP in fractions 7-9 when cells were lysed with Brij98. However, under these conditions, the amount of APP in TEMs was not altered in the presence of TSPAN8, suggesting that doxycycline rather than TSPAN8 caused the previously observed effect on APP. We conclude from this experiment that meprin $\beta$ and TSPAN8 are both located in TEMs where their interaction might occur. The cleavage of APP by meprin $\beta$ is also likely taking place within the TEMs.

Overall, we identified TSPAN8 as a novel non-proteolytic interaction partner of meprin $\beta$. We could show that this interaction takes place on the cell surface within TEMs (Figure 5). However, TSPAN8 did not influence the proteolytic activity of meprin $\beta$ towards fluorogenic peptides and did not alter the proteolytic processing of APP.

\section{Discussion}

Meprin $\beta$ is important for collagen processing and deposition (Kronenberg et al., 2010; Broder et al., 2013) and is associated with fibrotic conditions in Fra-2 transgenic mice (Biasin et al., 2014). As shed protease meprin $\beta$ is responsible for the cleavage of MUC2 in the intestine and subsequent detachment of the mucus layer critical for proper barrier function (Schütte et al., 2014). Only membrane bound meprin $\beta$ was shown to cleave APP at the $\beta$-secretase site reminiscent to BACE1 (Bien et al., 2012) and thus it is likely to play a role in Alzheimer's disease. This shows that meprin $\beta$ could be a promising pharmacological target for certain pathological conditions. To identify non-proteolytic interactions with regulatory
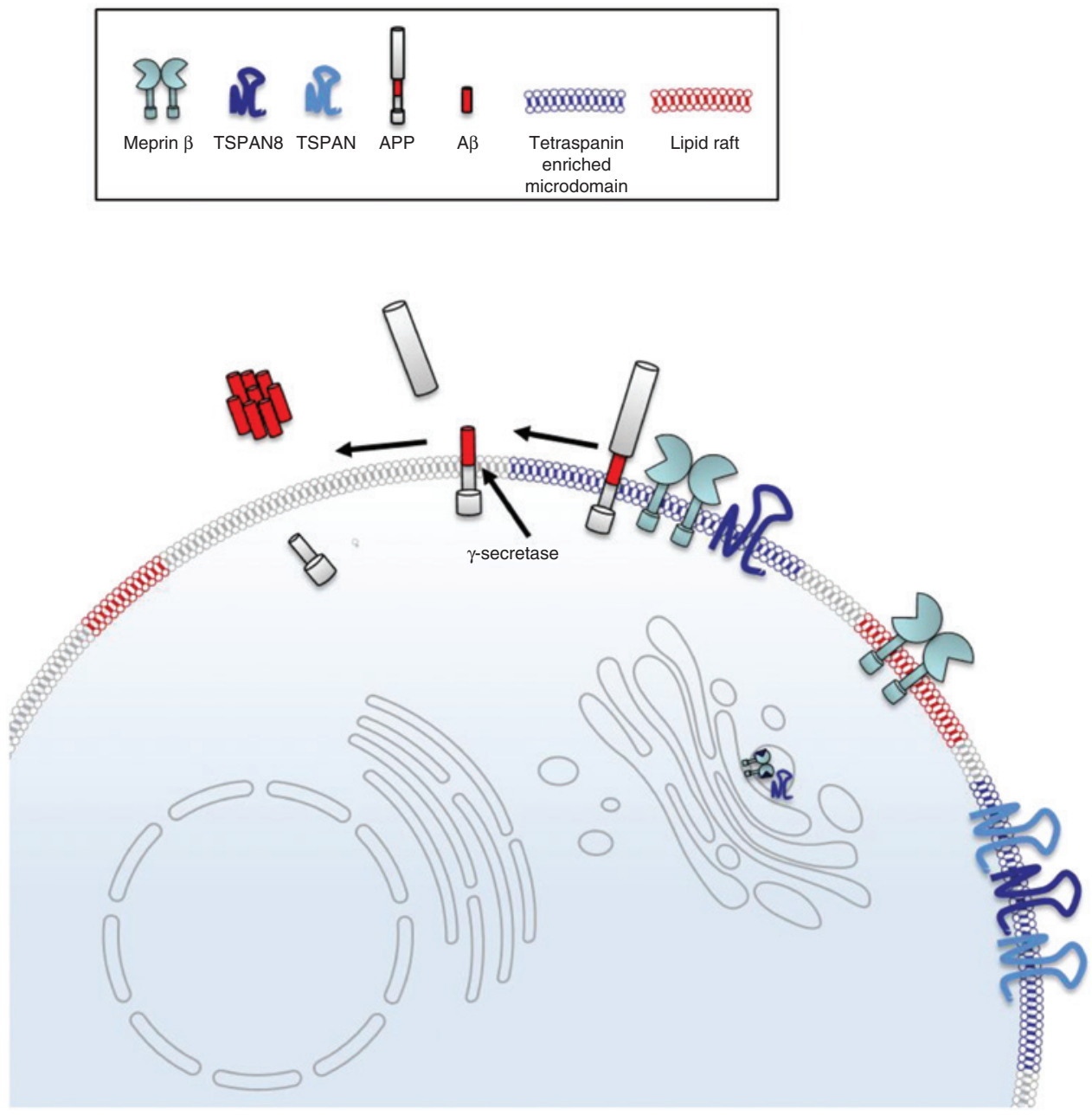

Figure 5: Potential model describing the interaction of meprin $\beta$ and TSPAN8.

TSPAN8 is a novel non-proteolytic interactor of meprin $\beta$. Meprin $\beta$ is found at the cell surface in both lipid rafts and tetraspanin-enriched microdomains (TEMs) whereas TSPAN8 is predominantly located within TEMs. The interaction of meprin $\beta$ and TSPAN8 therefore takes place inside the TEMs where cleavage of APP by meprin $\beta$ most likely occurs. Meprin $\beta$ acts as $\beta$-secretase on APP and upon additional cleavage by the $\gamma$-secretase short $A \beta$ peptides are released that are prone to aggregation. 
proteins involved in the activity or distribution of meprin $\beta$ we performed a yeast two-hybrid screen using a small intestinal cDNA library and meprin $\beta$ as bait. We identified TSPAN8 as a promising interaction partner in this screen. Members of the tetraspanin familiy are already known to interact with different metalloproteases, e.g. ADAMs (Gutierrez-Lopez et al., 2011; Haining et al., 2012; Prox et al., 2012). TSPAN8 for instance increases the cell surface expression of ADAM12, thereby promoting metastasis in esophageal cancer (Zhou et al., 2008). We confirmed the direct protein-protein interaction in human cells by a luciferase based protein complementation assay. Using a RFP dimerization assay, we localized the interaction of meprin $\beta$ and TSPAN8 to the cell surface. We then investigated if TSPAN8 has an impact on the proteolytic activity of meprin $\beta$. A quenched fluorogenic peptide cleavage assay revealed that the activity of meprin $\beta$ at the cell surface is unaltered in the presence of TSPAN8 in HEK293T cells. This was also the case when we inserted two point mutations (E1334A, E135A) in the LEL of TSPAN8, which would eventually fit into the active site cleft of meprin $\beta$ as it shows sequence homology to the propeptide. Additionally, shedding of meprin $\beta$ from the cell surface was not altered as its activity in the cell culture supernatant did not change when coexpressing TSPAN8. However, it could very well be that TSPAN8 changes the accessibility of meprin $\beta$ towards physiological proteinogenic substrates at the plasma membrane. For example, ADAM10 activity was shown to be enhanced by TSPAN12, leading to an increased processing of APP at the $\alpha$-secretase site (Xu et al., 2009). We therefore investigated the influence of TSPAN8 on the shedding activity of meprin $\beta$ towards APP. However, no difference in A $\beta$ production was detectable when TSPAN8 was present, concluding that the accessibility of APP for meprin $\beta$ is not changed. We first observed a decrease of $A \beta$ when inducing TSPAN8 expression by doxycycline or ATC. However, we could not confirm this change in $A \beta$ by transfection of TSPAN8. Therefore one has to be careful with the interpretation of APP processing when using doxycycline inducible cells. Furthermore, it is possible that other substrates might be affected in such a system.

It is well known that tetraspanins form TEMs together with other proteins such as integrins, $G$ protein-coupled receptors, growth factor receptors, but also cytosolic proteins (Charrin et al., 2009; Yanez-Mo et al., 2009). Thus, TEMs provide specialized signaling platforms and are involved in many cellular and biological processes (Hemler, 2003, 2005). Previously, it was shown that ADAM10 interacts with several TSPANC8 tetraspanins, an evolutionary conserved subgroup of tetraspanins exhibiting eight cysteines in their LEL, that regulate its maturation and cellular distribution (Haining et al., 2012). We therefore investigated the localization of meprin $\beta$ and TSPAN8 within the cell membrane. First, we performed cell surface biotinylation and confirmed the expression of both proteins at the cell membrane. However, we did not observe any differences in the amount of meprin $\beta$ at the cell surface as it was described for different ADAM proteases in the presence of tetraspanins (Zhou et al., 2008; Prox et al., 2012). Applying sucrose density gradient centrifugation we showed that meprin $\beta$ is not only found in lipid rafts but also in TEMs, together with TSPAN8. This corroborates our previous findings and proves that both proteins are colocalized in the same membrane subdomains. There was a recent example showing that tetraspanins, namely TSPAN5 and TSPAN15, have the ability to differentially regulate the membrane compartmentalization of ADAM10 (Jouannet et al., 2015). Interestingly, TSPAN8 did not alter the distribution of meprin $\beta$ within the cell membrane. We further analyzed whether TSPAN8 might influence the distribution of meprin $\beta$ substrates like APP. APP was mainly found in TEMs providing evidence that the processing of APP by meprin $\beta$ takes place in this environment. Similar observations were described in a recent study dealing with regulated intramembrane proteolysis (Chen et al., 2015). They observed that $\alpha$ - and $\beta$-secretases are able to form distinct complexes with $\gamma$-secretase and that tetraspanins were important for this association. This highlights the importance of protein networks for efficient processing of transmembrane substrates by different proteases (BeckerPauly and Rose-John, 2013). Further investigations will elucidate the function of meprin $\beta$ within this specialized environment. Potentially, meprin $\beta$ needs its interaction with TSPAN8 to meet so far unidentified substrates. Furthermore, detecting the interaction of the endogenous proteins would provide more information about its importance in certain cells or tissues. This would give a hint under which conditions this interaction takes place and what substrates might be affected in consequence. However, to this stage it is hard to show the interaction of endogenous meprin $\beta$ and TSPAN8 due to the lack of specific antibodies for TSPAN8. Additionally, it is likely that meprin $\beta$ and TSPAN8 synergize in the progression of different cancer types. TSPAN8 was found to be highly expressed in colon carcinoma, where it also promotes metastasis (Greco et al., 2010; Richardson et al., 2011; Yue et al., 2013). As meprin $\beta$ shows a high expression in the intestine and might be upregulated in colon cancer as well (Dietrich et al., 1996; Matters and Bond, 1999), this tumor type represents a promising condition to further investigate the interplay between meprin $\beta$ and TSPAN8. 


\section{Materials and methods}

\section{Chemicals}

All chemicals were of analytical grade and obtained from SigmaAldrich (St. Louis, MO, USA), Carl Roth (Karlsruhe, Germany), Merck (Darmstadt, Germany), Roche (Basel, Switzerland) and Thermo Fisher Scientific (Waltham, MA, USA).

\section{Split-ubiquitin yeast two-hybrid screen}

The split-ubiquitin yeast two-hybrid screen was performed in cooperation with Dualsystems Biotech (Schlieren, Switzerland). Shortly, human meprin $\beta$ cDNA was cloned into the bait vector pBT3-SUC and C-terminally fused to Cub-LexA-VP16. N-terminal NubG-tagged human small intestine library in pPR3-N was used as prey. Clones were selected, expanded and prey sequence traces were aligned and grouped. DNA sequences were translated in all three reading frames and searched against the Swissprot database using the BLASTX algorithm.

\section{Gaussia princeps luciferase-based protein complementation assay}

cDNA coding for human meprin $\beta$ and control, both lacking the stop codon, were cloned into the Gateway ${ }^{\odot}$ shuttle vector pENTR4 (\#17424, Addgene, Cambridge, MA, USA) using NotI/XhoI restriction sites. cDNA coding for human TSPAN8 in pDONR233 was a kind gift from Yves Jacob (Institute Pasteur, Paris, France). The cDNAs were then subcloned by homologous recombination into the destination vectors pSPICA-C1 and C2 (kind gift from Yves Jacob). pSPICA-C1 and $\mathrm{C} 2$ contain complementary fragments of Gaussia princeps luciferase located 3' of the Gateway ${ }^{\odot}$ recombination sites (Cassonnet et al., 2011). All coding sequences were verified by DNA sequencing (Gatc Biotech, Konstanz, Germany).

Luciferase complementation assay was performed as described previously (Cassonnet et al., 2011). Briefly, HEK293T cells were seeded at a density of $1 \times 10^{5}$ cells $/ \mathrm{ml}$ in $500 \mu \mathrm{l} /$ well of a 24-well plate. The following day, cells were transfected with plasmid DNA. Cells always received a combination of two plasmids, one coding for the C-terminal part of Gaussia princeps luciferase and one coding for the N-terminal part of Gaussia princeps luciferase, each fused to a protein of interest or as an empty control. On the second day after transfection, cells were washed, lysed in $100 \mu \mathrm{l}$ passive lysis buffer (\#E1941, Promega, Mannheim, Germany) for $15 \mathrm{~min}$ at RT, and then transferred, in triplicates of $20 \mu \mathrm{l}$ each, to a white 96-well bioluminescence plate. Coelenterazine was added and bioluminescence was detected using a Glomax plate injector system (Promega). Normalized luciferase ratio (NLR) was calculated as described in (Cassonnet et al., 2011). A NLR above 3.5 was considered a specific interaction. All experiments were $n=3$.

\section{Red fluorescent protein dimerization assay}

Destination vectors with RFP-A and RFP-B located $3^{\prime}$ of the Gateway ${ }^{\circ}$ recombination sites were created by deleting the Gaussia princeps luciferase fragment from pSPICA-C1 using site directed mutagenesis and inserting the restriction enzyme sites for AgeI and PacI. cDNA coding for RFP-A and RFP-B was amplified by PCR from pDDRFPA1B1-DEVD [\#36294, Addgene; original vectors are described in (Alford et al., 2012)] and then cloned into the destination vector using AgeI/PacI restriction sites. Homologous recombination was then performed with the entry vectors as described above.

HEK293T cells were seeded at a density of $2.5 \times 10^{4}$ cells $/ \mathrm{ml}$ in $\mu$-dishes (Ibidi, Martinsried, Germany). The following day, cells were transfected with plasmid DNA. Cells always received a combination of two plasmids, one coding for RFP-A and one coding for RFP-B, each fused to a protein of interest or as an empty control. On the second day after transfection, cells were imaged at an Olympus FluoView 1000 confocal microscope.

\section{Generation of HEK293T cells inducible for TSPAN8}

cDNA coding for TSPAN8 with a C-terminal myc tag was amplified by PCR and inserted into pINDUCER10 (Meerbrey et al., 2011) (kind gift from Jörg Müller, Institute of Molecular Cell Biology, Jena, Germany) using the AgeI/MluI restriction sites. The resulting plasmid was verified by sequencing (Gatc Biotech).

HEK293T cells were transfected with the plasmid. Cells were selected for plasmid uptake by culturing them in the presence of 2 $\mu \mathrm{g} / \mathrm{ml}$ puromycin. Cells were tested for tetraspanin expression in the presence and absence of $80 \mu \mathrm{g} / \mathrm{ml}$ doxycycline by immunoblotting of cell lysates.

\section{Site-directed mutagenesis for generation of TSPAN8 mutant}

Human wild-type cDNA of myc-tagged TSPAN8 in pcDNA3.1 was used as template for the generation of TSPAN8_E133A_E135A. Following primers were designed:

5'-AAA GCT TTT GAG CGC CAC AGG GGC AAG TGC AAA ACA ATT CCA GGA AGC CAT AAT T-3'

3'-AAT TAT GGC TTC CTG GAA TTG TTT TGC ACT TGC CCC TGT GGC GCT CAA AAG CTT T-5'

Mutagenesis was performed using the QuikChange ${ }^{\circledast}$ II XL SiteDirected Mutagenesis Kit (AgilentTechnologies, Santa Clara, CA, USA) according to the manufacturer's instructions. Correctness of mutations was proven by sequencing (Gatc Biotech).

\section{Cell culture, transient transfection and induction of HEK293T cells}

TSPAN8 inducible HEK293T cells were cultured in DMEM + GlutaMAX $^{\text {тм }}$-I (Life Technologies, Carlsbad, CA, USA) supplemented with $10 \%$ fetal bovine serum (Invitrogen) and $2 \mu \mathrm{g} / \mathrm{ml}$ puromycin. Transient transfection was performed using polyethylenimine $(1 \mathrm{mg} /$ $\mathrm{ml}$ in $\mathrm{ddH}_{2} \mathrm{O}$, Polysciences, Eppelheim, Germany) and following constructs: pcDNA3.1 (mock), hmeprin $\beta$ in pSG5, hAPP695 in pCIneo, hTSPAN8 in pcDNA3.1 and hTSPAN8_E133A_E135A in pcDNA3.1. For the induction of TSPAN8 expression, cells were treated with $100 \mathrm{ng} /$ $\mathrm{ml}$ to $80 \mu \mathrm{g} / \mathrm{ml}$ doxycycline or $100 \mathrm{ng} / \mathrm{ml}$ anhydrotetracycline (ATC) $24 \mathrm{~h}$ upon transfection. 


\section{Cell lysis, SDS-PAGE, and Western blot analysis}

Cell lysis, SDS-PAGE and Western blot analysis was performed as described previously (Arnold et al., 2015). Following antibodies were used for detection of protein in lysates: anti-GAPDH (2118; Cell Signaling Technology, Danvers, MA, USA), anti-actin (A2066; Sigma-Aldrich), anti-myc (9B11, 2276; Cell Signaling Technology), anti-meprin $\beta$ (polyclonal antibody, generated against a peptide of the ectodomain), anti-APP (N) (polyclonal antibody directed against N-terminus of APP; Thermo Fisher Scientific), anti-APP (C) (B63.2, polyclonal antibody directed against the C-terminus of APP), antiCD9 (SC-13118; Santa Cruz, Dallas, TX, USA), anti-Flotillin 2 (SC28320; Santa Cruz). Supernatants were used for analysis of SAPP $\alpha$ and A $\beta$ (6E10, SIG-39320; Covance, Princeton, NJ, USA).

\section{Meprin $\beta$ activity assay}

Determination of meprin $\beta$ proteolytic activity was performed using the specific quenched fluorogenic substrate (mca)-EDEDED-(K- $\varepsilon$ dnp) (Broder and Becker-Pauly, 2013; Jäckle et al., 2015). Culture medium and cell suspension with $50 \mu \mathrm{m}$ substrate were used for this assay. Changes in fluorescence intensity were measured every $30 \mathrm{~s}$ for $2 \mathrm{~h}$ at $37^{\circ} \mathrm{C}$ using the fluorescent spectrometer Infinite F200 PRO (Tecan, Maennedorf, Switzerland) with an excitation wavelength of $320 \mathrm{~nm}$ and detection of emission at $405 \mathrm{~nm}$.

dnp: 2,4-dinitrophenol; mca: (7-methyloxycoumarin-4-yl) acetyl.

\section{Molecular modeling}

The crystal structure of the extracellular domain of meprin $\beta$ (PDB accession number $4 \mathrm{GWN}$ ) was used with modeled epidermal growth factor (EGF)-like domain and transmembrane helix fused to it (Arolas et al., 2012). The large extracellular loop (LEL) of TSPAN8 was modeled using swiss-modeler (Biasini et al., 2014) where the LEL of CD81 was used as template (PDB accession number 1IV5). Meprin $\beta$ and TSPAN8 were positioned manually using UCSF Chimera (Pettersen et al., 2004) which was also used for the generation of molecular images.

\section{Cell surface primary amine biotinylation}

HEK293T cells were transfected with meprin $\beta$ and $24 \mathrm{~h}$ later TSPAN8 expression was induced. Primary amine biotinylation was performed as described previously (Arnold et al., 2015).

\section{Isolation of detergent resistant membrane fractions by sucrose density gradient centrifugation}

HEK293T cells were transfected with meprin $\beta$ and $24 \mathrm{~h}$ later TSPAN8 expression was induced. Alternatively, cells were transiently transfected with both meprin $\beta$ and TSPAN8. Next day, cells were harvested and lysed in $1 \mathrm{ml}$ TNE buffer ( $25 \mathrm{~mm}$ Tris, $150 \mathrm{~mm} \mathrm{NaCl}, 5 \mathrm{~mm}$ EDTA, complete protease inhibitor) for $30 \mathrm{~min}$ at $4^{\circ} \mathrm{C}$. For the isolation of lipid rafts the lysis buffer contained $1 \%$ Triton X-100 whereas for preservation of tetraspanin-enriched microdomains (TEMs) $1 \%$ Brij98 was added to the lysis buffer. Sucrose gradient preparation, centrifugation and analysis of the corresponding fractions were performed as described previously (Ebsen et al., 2015).

Acknowledgments: The authors thank Björn Rabe (Kiel, Germany) for providing the CD9 antibody, Xavier GomisRüth (Barcelona, Spain) for the pdb file of membranebound dimeric meprin $\beta$ and Yves Jacob (Paris, France) as well as Jörg Müller (Jena, Germany) for providing plasmids. This work was supported by the SFB877 'Proteolysis as a Regulatory Event in Pathophysiology' (project A9), grant BE 4086/2-1 (to C.B.-P.), grant PI 379/6-1 (to C.U.P.) and SFB841 (project C1, to D.S.-A.). W.A. is supported by KULeuven (C16/15/073), VIB, SAO (S\#14017) and the federal government (IAP P7/16).

\section{References}

Alford, S.C., Abdelfattah, A.S., Ding, Y., and Campbell, R.E. (2012). A fluorogenic red fluorescent protein heterodimer. Chem. Biol. 19, 353-360.

Arnold, P., Schmidt, F., Prox, J., Zunke, F., Pietrzik, C., Lucius, R., and Becker-Pauly, C. (2015). Calcium negatively regulates meprin beta activity and attenuates substrate cleavage. FASEB J. 29, 3549-3557.

Arolas, J.L., Broder, C., Jefferson, T., Guevara, T., Sterchi, E.E., Bode, W., Stocker, W., Becker-Pauly, C., and Gomis-Ruth, F.X. (2012). Structural basis for the sheddase function of human meprin beta metalloproteinase at the plasma membrane. Proc. Natl. Acad. Sci. USA. 109, 16131-16136.

Becker-Pauly, C. and Rose-John, S. (2013). TNFalpha cleavage beyond TACE/ADAM17: matrix metalloproteinase 13 is a potential therapeutic target in sepsis and colitis. EMBO Mol. Med. 5, 902-904.

Biasin, V., Marsh, L.M., Egemnazarov, B., Wilhelm, J., Ghanim, B., Klepetko, W., Wygrecka, M., Olschewski, H., Eferl, R., Olschewski, A., et al. (2014). Meprin beta, a novel mediator of vascular remodelling underlying pulmonary hypertension. J. Pathol. 233, 7-17.

Biasini, M., Bienert, S., Waterhouse, A., Arnold, K., Studer, G., Schmidt, T., Kiefer, F., Cassarino, T.G., Bertoni, M., Bordoli, L., et al. (2014). SWISS-MODEL: modelling protein tertiary and quaternary structure using evolutionary information. Nucleic Acids Res. 42, W252-258.

Bien, J., Jefferson, T., Causevic, M., Jumpertz, T., Munter, L., Multhaup, G., Weggen, S., Becker-Pauly, C., and Pietrzik, C.U. (2012). The metalloprotease meprin $\beta$ generates amino terminal-truncated amyloid $\beta$ peptide species. J. Biol. Chem. 287, 33304-33313.

Broder, C. and Becker-Pauly, C. (2013). The metalloproteases meprin alpha and meprin beta: unique enzymes in inflammation, neurodegeneration, cancer and fibrosis. Biochem. J. 450, 253-264. Broder, C., Arnold, P., Vadon-Le Goff, S., Konerding, M.A., Bahr, K., Muller, S., Overall, C.M., Bond, J.S., Koudelka, T., Tholey, 
A., et al. (2013). Metalloproteases meprin $\alpha$ and meprin $\beta$ are $\mathrm{C}$ - and $\mathrm{N}$-procollagen proteinases important for collagen assembly and tensile strength. Proc. Natl. Acad. Sci. USA. 110, 14219-14224.

Cassonnet, P., Rolloy, C., Neveu, G., Vidalain, P.O., Chantier, T., Pellet, J., Jones, L., Muller, M., Demeret, C., Gaud, G., et al. (2011). Benchmarking a luciferase complementation assay for detecting protein complexes. Nat. Methods 8, 990-992.

Charrin, S., Jouannet, S., Boucheix, C., and Rubinstein, E. (2014). Tetraspanins at a glance. J. Cell Sci. 127, 3641-3648.

Charrin, S., le Naour, F., Silvie, O., Milhiet, P. E., Boucheix, C., and Rubinstein, E. (2009). Lateral organization of membrane proteins: tetraspanins spin their web. Biochem. J. 420, 133-154.

Chen, A.C., Kim, S., Shepardson, N., Patel, S., Hong, S., and Selkoe, D.J. (2015). Physical and functional interaction between the $\alpha$ - and $\gamma$-secretases: a new model of regulated intramembrane proteolysis. J. Cell Biol. 211, 1157-1176.

Dietrich, J.M., Jiang, W., and Bond, J.S. (1996). A novel meprin $\beta^{\prime}$ mRNA in mouse embryonal and human colon carcinoma cells. J. Biol. Chem. 271, 2271-2278.

Ebsen, H., Lettau, M., Kabelitz, D., and Janssen, O. (2015). Subcellular localization and activation of ADAM proteases in the context of FasL shedding in T lymphocytes. Mol. Immunol. 65, 416-428.

Greco, C., Bralet, M.P., Ailane, N., Dubart-Kupperschmitt, A., Rubinstein, E., Le Naour, F., and Boucheix, C. (2010). E-cadherin/p120-catenin and tetraspanin Co-029 cooperate for cell motility control in human colon carcinoma. Cancer Res. 70, 7674-7683.

Gutierrez-Lopez, M.D., Gilsanz, A., Yanez-Mo, M., Ovalle, S., Lafuente, E.M., Dominguez, C., Monk, P.N., Gonzalez-Alvaro, I., Sanchez-Madrid, F., and Cabanas, C. (2011). The sheddase activity of ADAM17/TACE is regulated by the tetraspanin CD9. Cell Mol. Life Sci. 68, 3275-3292.

Hahn, D., Pischitzis, A., Roesmann, S., Hansen, M.K., Leuenberger, B., Luginbuehl, U., and Sterchi, E.E. (2003). Phorbol 12-myristate 13-acetate-induced ectodomain shedding and phosphorylation of the human meprinbeta metalloprotease. J. Biol. Chem. 278, 42829-42839.

Haining, E.J., Yang, J., Bailey, R.L., Khan, K., Collier, R., Tsai, S., Watson, S.P., Frampton, J., Garcia, P., and Tomlinson, M.G. (2012). The TspanC8 subgroup of tetraspanins interacts with A disintegrin and metalloprotease 10 (ADAM10) and regulates its maturation and cell surface expression. J. Biol. Chem. 287, 39753-39765.

Hemler, M.E. (2003). Tetraspanin proteins mediate cellular penetration, invasion, and fusion events and define a novel type of membrane microdomain. Annu. Rev. Cell Dev. Biol. 19, 397-422.

Hemler, M.E. (2005). Tetraspanin functions and associated microdomains. Nat. Rev. Mol. Cell Biol. 6, 801-811.

Jäckle, F., Schmidt, F., Wichert, R., Arnold, P., Prox, J., Mangold, M., Ohler, A., Pietrzik, C.U., Koudelka, T., Tholey, A., et al. (2015). Metalloprotease meprin $\beta$ is activated by transmembrane serine protease matriptase- 2 at the cell surface thereby enhancing APP shedding. Biochem. J. 470, 91-103.

Jefferson, T., Auf dem Keller, U., Bellac, C., Metz, V.V., Broder, C., Hedrich, J., Ohler, A., Maier, W., Magdolen, V., Sterchi, E., et al. (2013). The substrate degradome of meprin metalloproteases reveals an unexpected proteolytic link between meprin beta and ADAM10. Cell Mol. Life Sci. 70, 309-333.

Jouannet, S., Saint-Pol, J., Fernandez, L., Nguyen, V., Charrin, S., Boucheix, C., Brou, C., Milhiet, P.E., and Rubinstein, E. (2015). TspanC8 tetraspanins differentially regulate the cleavage of ADAM10 substrates, Notch activation and ADAM10 membrane compartmentalization. Cell Mol. Life Sci.

Kovalenko, O.V., Yang, X., Kolesnikova, T.V., and Hemler, M.E. (2004). Evidence for specific tetraspanin homodimers: inhibition of palmitoylation makes cysteine residues available for cross-linking. Biochem. J. 377, 407-417.

Kronenberg, D., Bruns, B.C., Moali, C., Vadon-Le Goff, S., Sterchi, E.E., Traupe, H., Bohm, M., Hulmes, D.J., Stocker, W., and Becker-Pauly, C. (2010). Processing of procollagen III by meprins: new players in extracellular matrix assembly? J. Invest. Dermatol. 130, 2727-2735.

Matters, G.L. and Bond, J. S. (1999). Expression and regulation of the meprin $\beta$ gene in human cancer cells. Mol. Carcinog. 25, 169-178.

Meerbrey, K.L., Hu, G., Kessler, J.D., Roarty, K., Li, M.Z., Fang, J.E., Herschkowitz, J.I., Burrows, A.E., Ciccia, A., Sun, T., et al. (2011). The pINDUCER lentiviral toolkit for inducible RNA interference in vitro and in vivo. Proc. Natl. Acad. Sci. USA. 108, 3665-3670.

Ohler, A., Debela, M., Wagner, S., Magdolen, V., and Becker-Pauly, C. (2010). Analyzing the protease web in skin: meprin metalloproteases are activated specifically by KLK4, 5 and 8 vice versa leading to processing of proKLK7 thereby triggering its activation. Biol. Chem. 391, 455-460.

Perneczky, R., Alexopoulos, P., and Kurz, A. (2014). Soluble amyloid precursor proteins and secretases as Alzheimer's disease biomarkers. Trends Mol. Med. 20, 8-15.

Pettersen, E.F., Goddard, T.D., Huang, C.C., Couch, G.S., Greenblatt, D.M., Meng, E.C., and Ferrin, T.E. (2004). UCSF Chimera - a visualization system for exploratory research and analysis. J. Comput. Chem. 25, 1605-1612.

Prox, J., Willenbrock, M., Weber, S., Lehmann, T., Schmidt-Arras, D., Schwanbeck, R., Saftig, P., and Schwake, M. (2012). Tetraspanin15 regulates cellular trafficking and activity of the ectodomain sheddase ADAM10. Cell Mol. Life Sci. 69, 2919-2932.

Richardson, M.M., Jennings, L.K., and Zhang, X.A. (2011). Tetraspanins and tumor progression. Clin. Exp. Metastasis. 28, 261-270.

Schütte, A., Ermund, A., Becker-Pauly, C., Johansson, M.E., Rodriguez-Pineiro, A.M., Backhed, F., Muller, S., Lottaz, D., Bond, J.S., and Hansson, G.C. (2014). Microbial-induced meprin $\beta$ cleavage in MUC2 mucin and a functional CFTR channel are required to release anchored small intestinal mucus. Proc. Natl. Acad. Sci. USA. 111, 12396-12401.

Sterchi, E.E., Stocker, W., and Bond, J.S. (2008). Meprins, membrane-bound and secreted astacin metalloproteinases. Mol. Aspects Med. 29, 309-328.

Xu, D., Sharma, C., and Hemler, M.E. (2009). Tetraspanin12 regulates ADAM10-dependent cleavage of amyloid precursor protein. FASEB J. 23, 3674-3681.

Yanez-Mo, M., Barreiro, O., Gordon-Alonso, M., Sala-Valdes, M., and Sanchez-Madrid, F. (2009). Tetraspanin-enriched microdomains: a functional unit in cell plasma membranes. Trends Cell Biol. 19, 434-446. 
Yanez-Mo, M., Gutierrez-Lopez, M.D., and Cabanas, C. (2011). Functional interplay between tetraspanins and proteases. Cell Mol. Life Sci. 68, 3323-3335.

Yue, S., Mu, W., and Zoller, M. (2013). Tspan 8 and CD151 promote metastasis by distinct mechanisms. Eur. J. Cancer. 49, 2934-2948.

Zhou, Z., Ran, Y.L., Hu, H., Pan, J., Li, Z.F., Chen, L.Z., Sun, L.C., Peng, L., Zhao, X.L., Yu, L., et al. (2008). TM4SF3 promotes esopha- geal carcinoma metastasis via upregulating ADAM12m expression. Clin. Exp. Metastasis. 25, 537-548.

Supplemental Material: The online version of this article (DOI: 10.1515/hsz-2016-0126) offers supplementary material, available to authorized users. 